Opt Commun. 2020 January 1; 454: . doi:10.1016/j.optcom.2019.124364.

\title{
Design of $45^{\circ}$ periscopic visual field expansion device for peripheral field loss
}

\author{
Hee-Jin Choi ${ }^{1}$, Eli Peli ${ }^{2}$, Minyoung Park ${ }^{1}$, Jae-Hyun Jung ${ }^{2,{ }^{*}}$ \\ ${ }^{1}$ Department of Physics and Astronomy, Sejong University, Seoul 05006, Korea \\ ${ }^{2}$ Schepens Eye Research Institute, Massachusetts Eye and Ear, Department of Ophthalmology, \\ Harvard Medical School, Boston, MA, USA
}

\begin{abstract}
Patients with visual field loss have difficulty in mobility due to collision with pedestrians/obstacles from the blind side. In order to retrieve the lost visual field, prisms which deflect the field from the blind to the seeing side, have been widely used. However, the deflection power of current clinical Fresnel prisms is limited to $\sim 30^{\circ}$ and only provides a $5^{\circ}$ eye scanning range to the blind side. This is not sufficient to avoid collision and results in increasing demands for a device with a higher power. In this paper, we propose a novel design and optimization of a higher power prism-like device (cascaded structure of mirror pairs filled with high refractive index) and verify enhanced expansion of up to $45^{\circ}$ in optical ray tracing and photorealistic simulations.
\end{abstract}

Individuals with visual field loss report collisions with other pedestrians/objects and tripping over obstacles, and are commonly not permitted to drive. All of these factors severely restrict their mobility and quality of life. Peripheral visual field loss, tunnel vision (severe peripheral field constriction to $<20^{\circ}$ of residual central field), maybe due to retinal diseases such as retinitis pigmentosa, choroideremia, and advanced glaucoma [1,2]. The loss of half of the visual field in both eyes on the same side (Homonymous hemianopia, $\mathrm{HH}$ ), which is a common peripheral field loss, can be caused by brain injuries such as stroke, trauma, or tumors [3-6].

Among various efforts to help the patients with visual field loss as described above, prism glasses have been long considered one of the simplest, most effective, and helpful devices for field expansion, shifting (deflecting) the field from the blind side (prism base side) to the seeing side of the patient with visual field loss. Throughout previous studies, peripheral prism glasses [4] to avoid confusions in the central visual field are established as an effective field expansion device for field loss patients in walking [2] and driving [7]. Regarding the concept of visual field expansion, it is obvious that larger deflection power can deliver more

*Corresponding author: jaehyun_jung@meei.harvard.edu.

Publisher's Disclaimer: This is a PDF file of an unedited manuscript that has been accepted for publication. As a service to our customers we are providing this early version of the manuscript. The manuscript will undergo copyediting, typesetting, and review of the resulting proof before it is published in its final citable form. Please note that during the production process errors may be discovered which could affect the content, and all legal disclaimers that apply to the journal pertain. 
visual information from farther blind side to the seeing side of patients with visual field loss, devices with higher prism power (deflection power).

Recently, Peli et al. [8] found that the collision risk with other walking pedestrian increases with eccentricity, and peaks at $45^{\circ}$. Thus, visual field expansion to $45^{\circ}$ is desirable to effectively reduce the risk of collision. However, current clinically available prism glasses provide only up to $\sim 30^{\circ}$ field expansion with $57 \Delta$ (prism diopter) Fresnel prisms [9]. In addition, supporting wider eye scanning range (up to $15^{\circ}$ range of most eye movements [10] into the blind side) and better image quality [9] are also desirable.

The main effect that restricts the optical power of conventional prism is the total internal reflection (TIR). The current clinically available $57 \Delta$ prism allows only $5^{\circ}$ eye scanning range into the blind side due to the TIR [6], which is far less than the maximal eye movements range of $15^{\circ}$ mentioned above. Although higher apex angle results in higher prism power, TIR limits the rated prism power (measured at the normal incidence) higher than $41^{\circ}$ as shown in Figs. 1(a) and (b).

Though it is possible to move the TIR limit farther by using angle of smaller or minimum deviation in the prism, it results in smaller field expansion [6]. The images seen through the prism when approaching the TIR limitation are highly distorted (minified) and dimmer [6]. Color dispersion in prisms lowers the image quality further as it reduces contrast [11]. When using Fresnel prisms to reduce the size and weight of high power prisms in current clinical prism glasses, light scattered at the non-imaging base surface further reduces image quality $[6,11]$. The reduced image quality may significantly affect hazard detection through the prism.

There is a need for a device which provides higher optical power, wider scanning range, and less distortion. Previously, we proposed a concept of higher power mirror prism-like element [9], which is a cascaded structure of mirror periscopic prism (MP). An MP deflects the light path through double reflections, and the device's (deflection) power is simply double the apex angle (the angle between mirrors) as shown in Figs. 1(c) and (d). The reflective deflection from an MP is free of refractive image degradations such as distortion, transmittance reduction, color dispersion, and TIR (Fig. 1d) [9]. Note that the MP does not have a physical base and apex, but we name them to match with the physical base and apex in the conventional prism and thus the deflection direction. The conceptual design in our previous work [9] has not achieved the practical goal of $45^{\circ}$ optical power and $60^{\circ}$ field of view $(\mathrm{FoV})$ due to the limitation that the sizes of mirrors increase in order to provide more optical power and FoV as shown in Fig. 1(d). An MP with higher power requires impractically longer mirrors.

In this paper, we analyze the requirements of the cascaded MP modules and propose a new MP design filed with polymethyl methacrylate (PMMA) to hold the structure and provide refractive power. The new MP design provides a higher deflection power of $45^{\circ}$ by combining the low refractive and high reflective deflection powers in practical sizes. It also provides FoV up to $\sim 57^{\circ}\left(42^{\circ}\right.$ seeing FoV and $15^{\circ}$ eye scanning range) for normal eye scanning in $\mathrm{HH}[5]$. 
We derive Eqs. (1)-(4) for the design of the cascaded MP modules with reflective deflection power, $\delta_{M}$. The main parameters of cascaded MP modules (Fig. 2) are the size of mirrors $\left(M_{n}\right)$ and the distance between MP modules and the center of the entrance pupil of the eye $\left(D_{n}\right)$. We denote the FoV of each module and the angle of the $1^{\text {st }}$ reflection from the mirror surface as $\theta 9$ and $\beta$ respectively. Since the MP modules abut each other in the cascade structure in order to prevent the blocking of light paths from neighboring modules, the derived equations also have a form of series equations. For patients with left $\mathrm{HH}$, the left side mirror of the $n^{\text {th }}$ MP module should be the right side mirror of the $(n+1)^{\text {th }}$ MP module as shown in Fig. 2. The relationship between the length of the mirror $\left(M_{n}\right)$ and the distance to the mirror $\left(D_{n}\right)$ is derived as follows.

$$
\begin{gathered}
\beta_{n+1}=\beta_{n}+\frac{\delta_{M n}}{2}-\theta_{n}, \\
D_{n+1}=D_{n}+\frac{\sin 2 \beta_{n}}{\sin \left(2 \beta_{n}-\theta_{n}\right)}, \\
\beta_{n+1}=\beta_{n}+\frac{\delta_{M n}}{2}-\theta_{n}, \\
M_{n+1}=D_{n} \frac{\sin \theta_{n} \sin \beta_{n} \sin 2\left(\beta_{n}-\theta_{n}\right)}{\sin \left(\beta_{n}-\theta_{n}\right) \sin \left(2 \beta_{n}-\theta_{n}\right) \sin \left(\beta_{n}-\theta_{n}-\frac{\delta_{M_{n}}}{2}\right)}
\end{gathered}
$$

Equation 4 also shows that a single MP module with $45^{\circ}$ prism power and $60^{\circ} \mathrm{FoV}$ requires an impractical $3.7 \mathrm{~m}$ mirror $\left(M_{2}\right.$ at $5 \mathrm{~mm}$ from the eye $\left(D_{2}\right)$ with $82.5^{\circ}$ of $\beta_{2}$. A cascade of MP modules is therefore required for practically small and safe design. We set design constraints that $M$ and $D$ should be less than $20 \mathrm{~mm}$ and larger than $16 \mathrm{~mm}$ (typical distance from the center of the entrance pupil of the eye to the back surface of a corrective lens [12]), respectively.

The shaded area in Fig. 3 shows the combinations of $\beta$ and $\Theta$ that satisfy the safety consideration of $D_{n+1} \geq D_{n}$ and physical consideration of $M_{n+1} \geq 0$ for various $\delta_{M}$ from $30^{\circ}$ to $45^{\circ}$. The relation to satisfy the consideration of $M_{n+1} \geq 0$ is $\sin \left(\beta_{n}-\theta_{n}-\left(\delta_{M_{n}} / 2\right)\right) \geq 0$ since all the other terms in Eq. (4) are positive. The graph shows that the angle of the $1^{\text {st }}$ reflection $(\beta)$ is restricted by the FoV in a single MP module to satisfy the above requirements.

We derived the restriction of FoV ( $\theta$ ) from the practical constraint of $M_{n+1} \leq 20 \mathrm{~mm}$ as shown in Fig. 4 with the same range of $\delta_{M}$. The analysis shows that the size of mirror $M$ is increasing steeply with $\theta$. If $\delta_{M}$ increases, the size of mirror $M$ should be much larger than the practical limitation. Thus, we found that the FoV of a single MP should be less than $15^{\circ}$ to meet the practical limit even if $\delta_{M}$ is $30^{\circ}$. Therefore, at least four MP modules are necessary to achieve the overall field of $\approx 60^{\circ}$. 
Though the basic design rule has been derived through the analyses above for an MP with $\delta_{M}=30^{\circ}$, it is still necessary to further increase $\delta_{M}$ while keeping the practical limitation of $M_{n+1} \leq 20 \mathrm{~mm}$ and the safety requirement of $D_{n} \geq 16 \mathrm{~mm}$, as noted above. To achieve that goal, we propose a design where the cascaded MP modules have the same total deflection power $\delta$ from differently distributed reflective $\left(\delta_{M}\right)$ and refractive $\left(\delta_{R}\right)$ deflection powers. We filled the space between mirrors with a refractive medium (PMMA, refractive index $=$ 1.49) which provides additional flexibility of design by allowing us to control $\delta_{R}$.

The main idea is giving the MPs lower $\delta_{M}$ in order to limit the mirror sizes. Then, the cascade structure enforcing an increase of the mirror size $\left(M_{n+1}<M_{n}\right)$ makes the PMMA between the mirrors form a prism shape at the exit window of the MPs with $\delta_{R}$. Additional control of $\delta_{R}$ is available by changing the mirror size $M_{n}$ slightly. The effect on the image quality from the refraction is negligibly small due to the small angle of incidence at the surface [4]. With these principles, we designed a device with FoV of $57.1^{\circ}$ and deflection power of approximately $45^{\circ}$ while satisfying the practical and safety considerations. The parameters have been slightly different from the preliminary calculations based on Eqs. (1)-(4) as the $\delta_{R}$ has been added by iterative changes of mirror sizes, and all MP modules satisfy both the dimensional and optical requirements. Table 1 shows the detailed parameters of four cascaded MP modules. Note that it is possible to achieve a higher refractive power $\delta_{R}$ as $M_{n}$ is increased due to the cascaded structure. Thus, it is important to adjust the inclination angle of the exit window to have less refractive power $\delta_{R}$ in order to maintain the total deflection power of $45^{\circ}$.

We performed a ray-tracing simulation using LightTools (Synopsis, Mountain View, CA) to verify the field expansion function of the proposed design. The dimension of the designed MP is $36 \mathrm{~mm} \times 16 \mathrm{~mm} \times 8 \mathrm{~mm}(\mathrm{~W} \times \mathrm{D} \times \mathrm{H})$, respectively. The calculated total deflection powers $\delta$ of each module are shown in Fig. 5. Though there are small tunnel scotomas [13] between modules, their effects are negligible $\left(<0.1^{\circ}\right)$ at the distances of objects of interest in mobility. The variation in deflection powers between modules due to the additional refractive prism structure $\left(\delta_{R}\right)$ was about $1^{\circ}$.

We illustrated the image quality and field expansion in the perceived scene. Since there is no way to visualize and compare the perceived scene by a patient with $\mathrm{HH}$, the effects of MP and conventional Fresnel prism with vertical size of $8 \mathrm{~mm}$ (conventional peripheral prisms $[5,6])$ were compared using photorealistic rendering with a virtual pinhole camera in LightTools. We used a pinhole camera simulation to clearly illustrate the size of FoV and amount of the shift without blur effects in the boundaries of the MP with finite pupil. In the simulation, a flat virtual image wall has been set at $6 \mathrm{~m}$ from the flat virtual camera sensor (simulated eye) to cover $120^{\circ}$ of the visual field. At the virtual wall, an image composed of a pattern of vertical lines (Fig. 6(a)) was mapped to illustrate the total deflection power and the transmittance for the designed MP shown in Fig. 6(b). We also modeled a conventional $57 \Delta$ Fresnel prism in PMMA with a $38.5^{\circ}$ apex angle, and each segment had a $0.35 \mathrm{~mm}$ width for comparison. The size of the $57 \Delta$ Fresnel prism was $16.4 \mathrm{~mm}$ in order to cover $28.8^{\circ} \mathrm{FoV}(57 \Delta)$ in the seeing side and $15^{\circ}$ in the blind side. 
Figure 6 illustrates the differences in visual field expansion and image quality between the MP and the Fresnel prism. A perceived view through the MP is shown in Fig. 6(b). The simulation shows an expansion of $45^{\circ}$ without distortion and high transmittance. About $15^{\circ}$ of eye scanning range enables the MP to provide a total FoV of $57^{\circ}$ as designed. In contrast, conventional Fresnel prism distortion increases towards the TIR, which blocks the shifted view beyond $5^{\circ}$ eccentricity. Thus, the Fresnel prism is only providing a visual field expansion of $29^{\circ}$ with a FoV of about $34^{\circ}$ (right $29^{\circ}$ in seeing FoV and left $5^{\circ}$ for additional eye scanning range). The perceived scene through the Fresnel prism has lower transmittance near the TIR (e.g., an orange bar as an indicator of $40^{\circ}$ left). Since the height of the device's entrance pupil (toward the eye) and exit pupil (outward) are the same, the smaller angular size of the exit pupil than the entrance pupil due to its larger distance results in the protrusion scotoma as shown by black areas in Fig. 6(b). Different protrusion in each module (different distance to the exit pupil among modules) resulted in different vertical angular size of the perceived image.

To illustrate that the proposed MP would enable patients to detect possible collision we simulated images with pedestrians in the blind side (Fig. 7). A scene with three walking pedestrians at eccentricities of $29^{\circ}, 41^{\circ}$, and $51^{\circ}$ as shown in Fig. 7 (a) is simulated through the MP (Fig. 7(b) and compared with the simulated scene through the current $57 \Delta$ Fresnel prism(Fig. 7(c).

As illustrated in Fig. 7(b), a left $\mathrm{HH}$ patient with an MP could detect the $1^{\text {st }}$ and $2^{\text {nd }}$ pedestrians at left $29^{\circ}$ and $41^{\circ}$ from the primary position of gaze and would be able to see the $3^{\text {rd }}$ pedestrian at $51^{\circ}$ left with eye scanning into the blind side. Thus, we can expect that the patient can effectively evade collisions with the help of the expanded view through the proposed MP design. In contrast, the conventional 57 $\Delta$ Fresnel prism in Fig. 7(c) only covers the $1^{\text {st }}$ pedestrian at the primary position of gaze and would not show clear views of the farther $2^{\text {nd }}$ or $3^{\text {rd }}$ pedestrians, even with the eye scanning into the blind side due to the severe distortion followed by TIR and spurious reflection.

As a conclusion, we designed the MP and verified with ray tracing and photorealistic rendering so that our design could extend the visual field of patients with $\mathrm{HH}$ with a deflection power of $45^{\circ}$ to detect the peak collision risk with other pedestrians far beyond the range of the current clinically available prism. The MP provides much better image quality, which may improve the detection performance. The MP also may be applied to patients with other types of visual field loss, such as tunnel vision [5,6]. The potential functionality of the device was demonstrated. The protrusion scotoma (upper and lower black areas in Fig. 6(b) and 7(b)) due to the different angular size of the entrance and exit pupils of the device can be resolved in further developments.

\section{Acknowledgments}

Funding Sources. This research was supported by National Institute of Health (R01EY23385) and Basic Science Research Program through the National Research Foundation of Korea funded by the Ministry of Education (2018R1D1A1B07049563) 


\section{References}

1. Apfelbaum $\mathrm{H}$ and Peli E, "Tunnel vision prismatic field expansion: Challenges and requirements," Transl. Vis. Sci. Technol. 4, 8 (2015).

2. Qiu C, Jung J-H, Tuccar-Burak M, Spano L, Goldstein R, and Peli E, "Measuring Pedestrian Collision Detection With Peripheral Field Loss and the Impact of Peripheral Prisms," Transl. Vis. Sci. Technol. 7, 1 (2018).

3. Cohen JM, "An overview of enhancement techniques for peripheral field loss," J. Am. Optom. Assoc. 64, 60 (1993). [PubMed: 8454830]

4. Peli E, "Field expansion for homonymous hemianopia by optically induced peripheral exotropia," Optom. Vis. Sci. 77, 453 (2000). [PubMed: 11014672]

5. Apfelbaum HL, Ross NC, Bowers AB, and Peli E, "Considering apical scotomas, confusion, and diplopia when prescribing prisms for homonymous hemianopia," Transl. Vis. Sci. Technol. 2, 2 (2013).

6. Jung J-H and Peli E, "Impact of high power and angle of incidence on prism corrections for visual field loss," Opt. Eng. 53, 061707 (2014).

7. Houston KE, Peli E, Goldstein RB, and Bowers AR, "Driving with hemianopia VI: Peripheral prisms and perceptua-motor training improve blind-side detection in a driving simulator," Transl. Vis. Sci. Technol. 7, 5 (2018).

8. Bahill AT, Adler D, and Stark L, "Most naturally occurring human saccades have magnitudes of 15 degrees or less," Investigative ophthalmology 14, 468 (1975). [PubMed: 1132942]

9. Peli E, Bowers AR, Keeney K, and Jung J-H, "High power prismatic devices for oblique peripheral prisms," Optom. Vis. Sci. 93, 521 (2016). [PubMed: 26866438]

10. Peli E, Apfelbaum H, Berson EL, and Goldstein RB, "The risk of pedestrian collisions with peripheral visual field loss," J. Vis. 16, 1 (2016).

11. Katz M, "Visual acuity through Fresnel, refractive, and hybrid diffractive/refractive prisms," Optometry 75, 503 (2004). [PubMed: 15372787]

12. Gross H, Blechinger F, and Achtner B, "Human Eye," in Handbook of Optical Systems, Volume 4: Survey of Optical Instruments, Gross H, ed. (Wiley-VCH Verlag GmBH \& Co. KGaA Weinheim, Germany, 2008).

13. Jung J-H and Peli E, "Field Expansion for Acquired Monocular Vision Using a Multiplexing Prism," Optom. Vis. Sci. 95, 814 (2018). [PubMed: 30169357] 


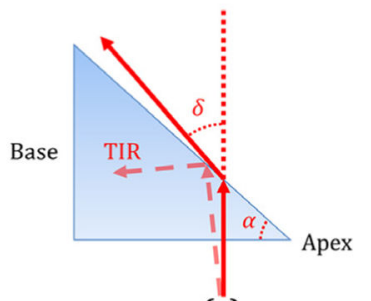

(a)

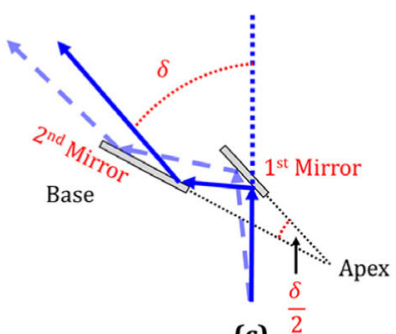

(c)

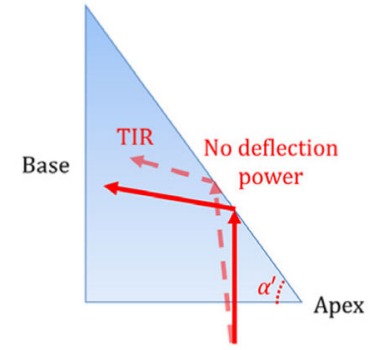

(b)

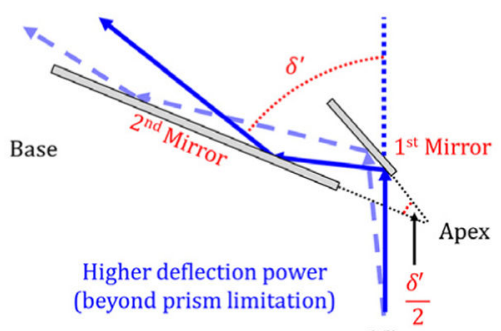

(d)

Fig. 1.

Limitation of conventional and reflective prisms. (a) Limitation of a conventional prism. In a conventional PMMA prism with an apex angle (a), maximal deflection power $(\delta)$ is $\sim 41^{\circ}$ (50\% transmittance) with no eye scanning range toward the blind side of $\mathrm{HH}$ (dashed arrows). (b) Due to the TIR, higher apex angle (a) does not result in higher deflection power even at the normal incidence. (c) Principle of a reflective deflection in an MP. The reflective deflection power $(\delta)$ of an MP based on double reflection is double the apex angle $(\delta / 2)$. (d) The MP can provide higher deflection power $(\delta)$ with a higher apex angle $(\delta / 2)$ with no limitation of TIR but requires longer mirrors. 


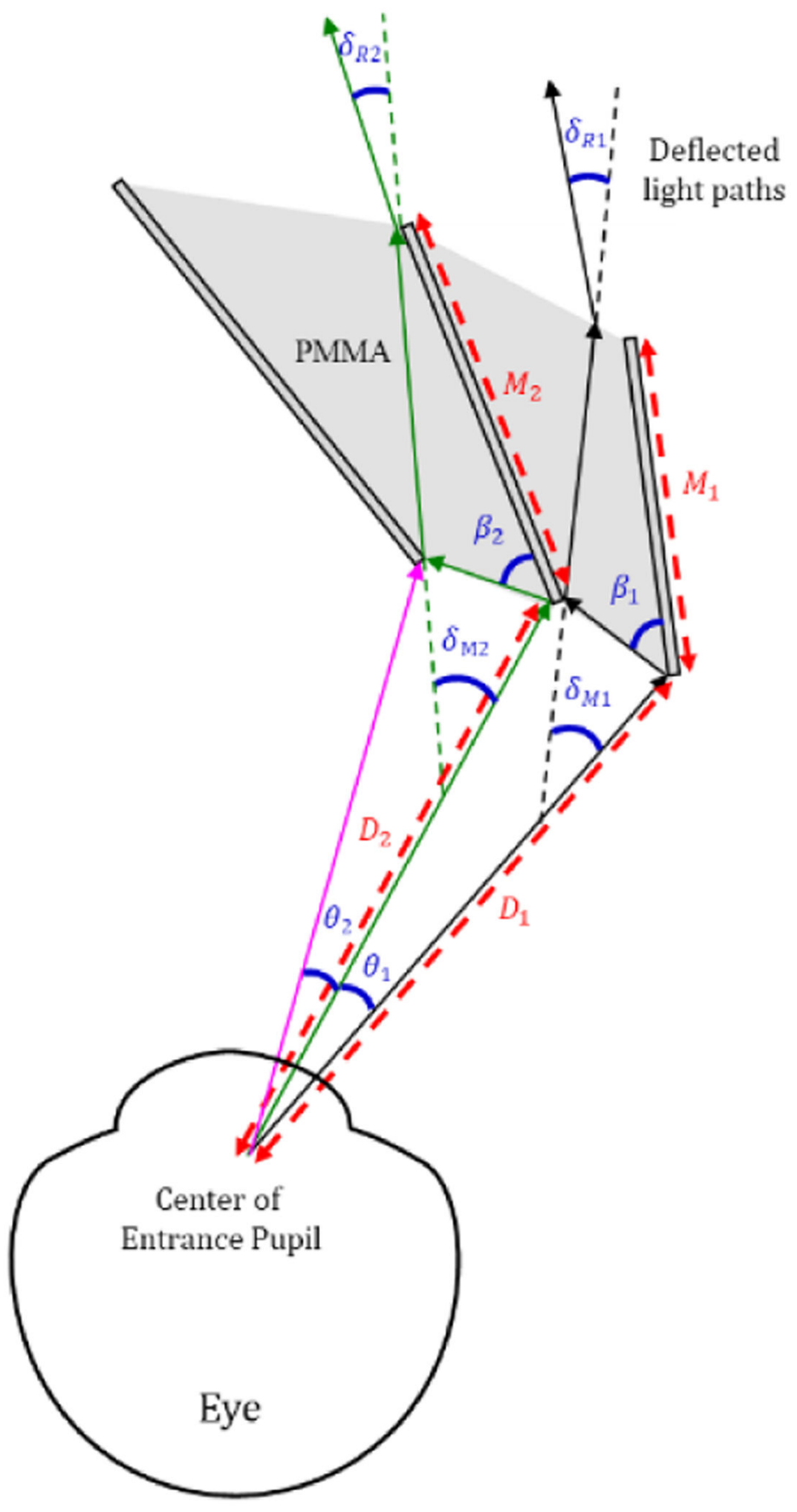

Fig. 2.

Parameters of cascaded MP modules. $D_{n}$ is the distance between the edge of the mirror and the entrance pupil of the eye, and $\mathrm{Mn}$ the size of the nth mirror. The FoV of each module is $\theta$. Parameters $\beta, \delta_{M}$, and $\delta_{R}$ are the angle of the 1 st reflection from the mirror surface, reflective deflection power, and refractive deflection power, respectively. 


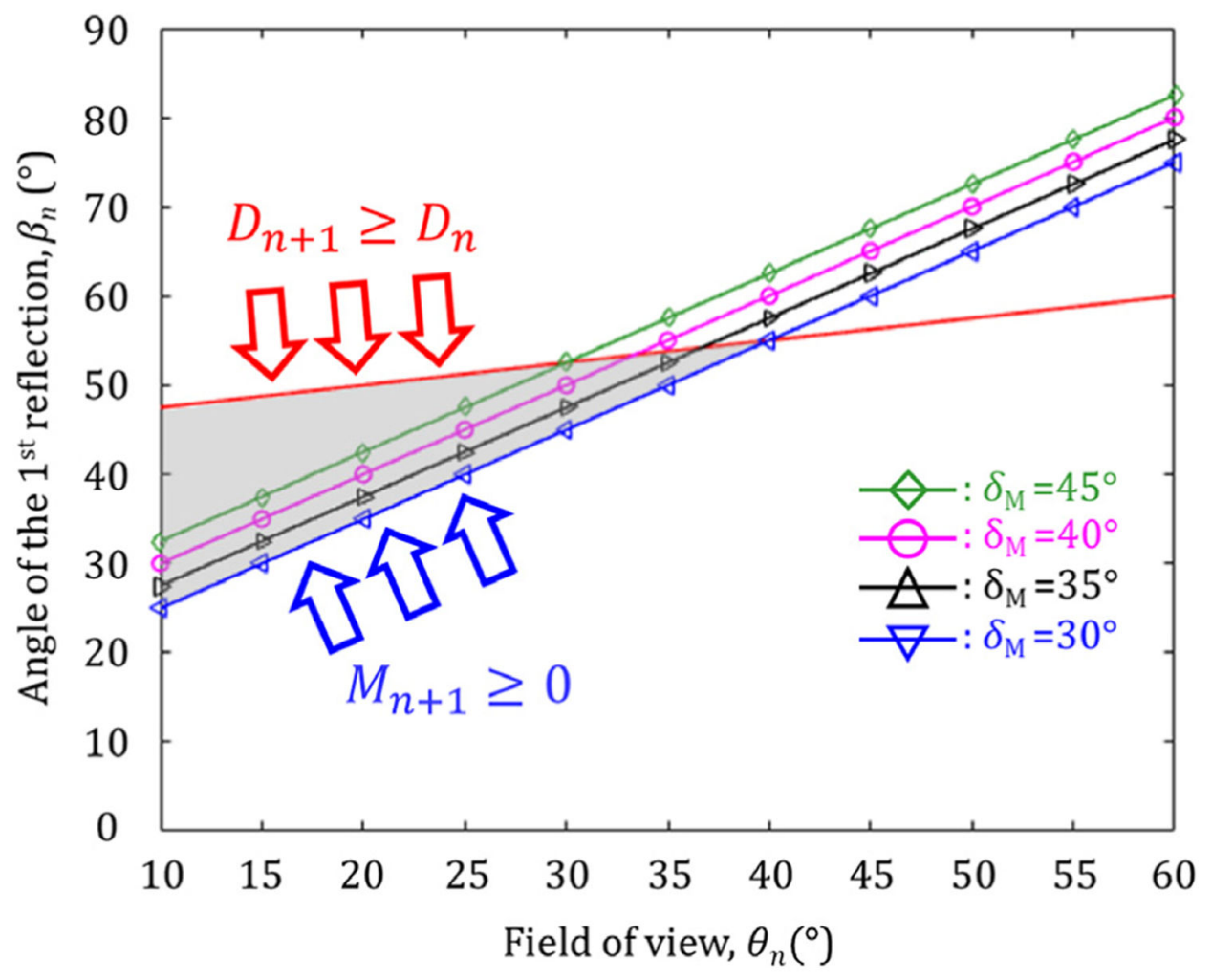

Fig. 3.

Relations between $\beta$ and $\Theta$. The shaded area satisfies both the safety $\left(D_{n+1} \geqslant D_{n}\right)$ and physical $\left(M_{n+1} \geqslant 0\right)$ considerations. 


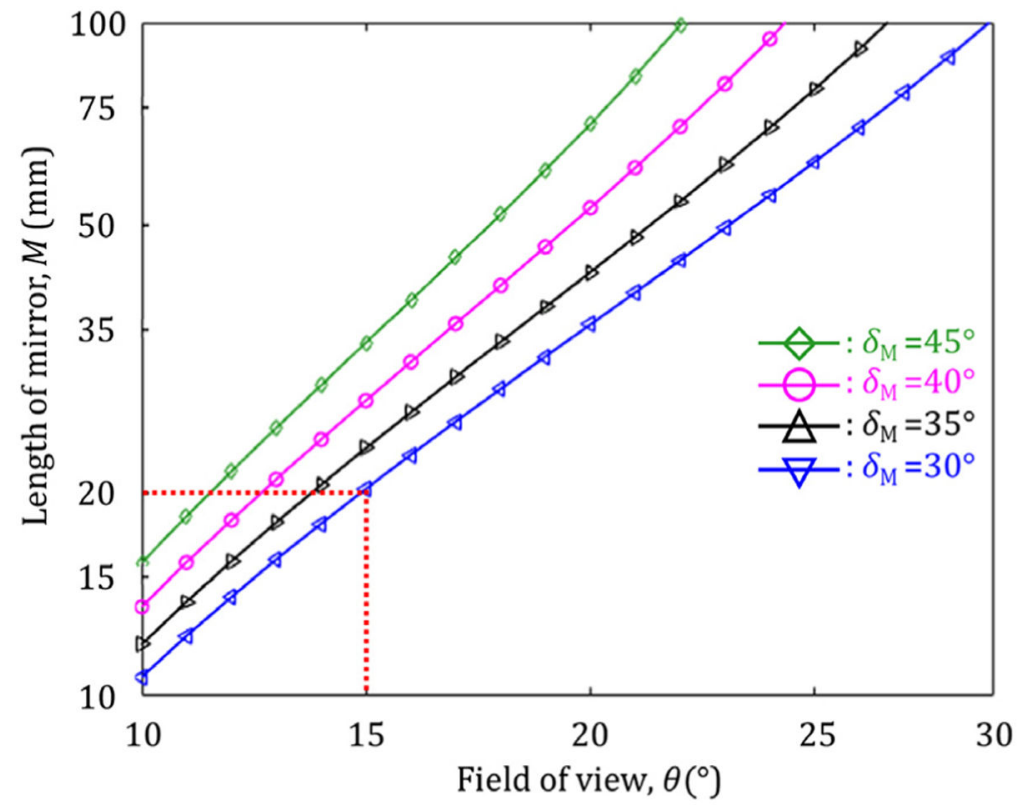

Fig. 4.

Practical limitation $\left(M_{n+1} \leqslant 20 \mathrm{~mm}\right)$ for a single MP, which leads to a single MP with less than $15^{\circ} \mathrm{FoV}(\theta)$ Note that $\mathrm{M}$ is in log scale. 


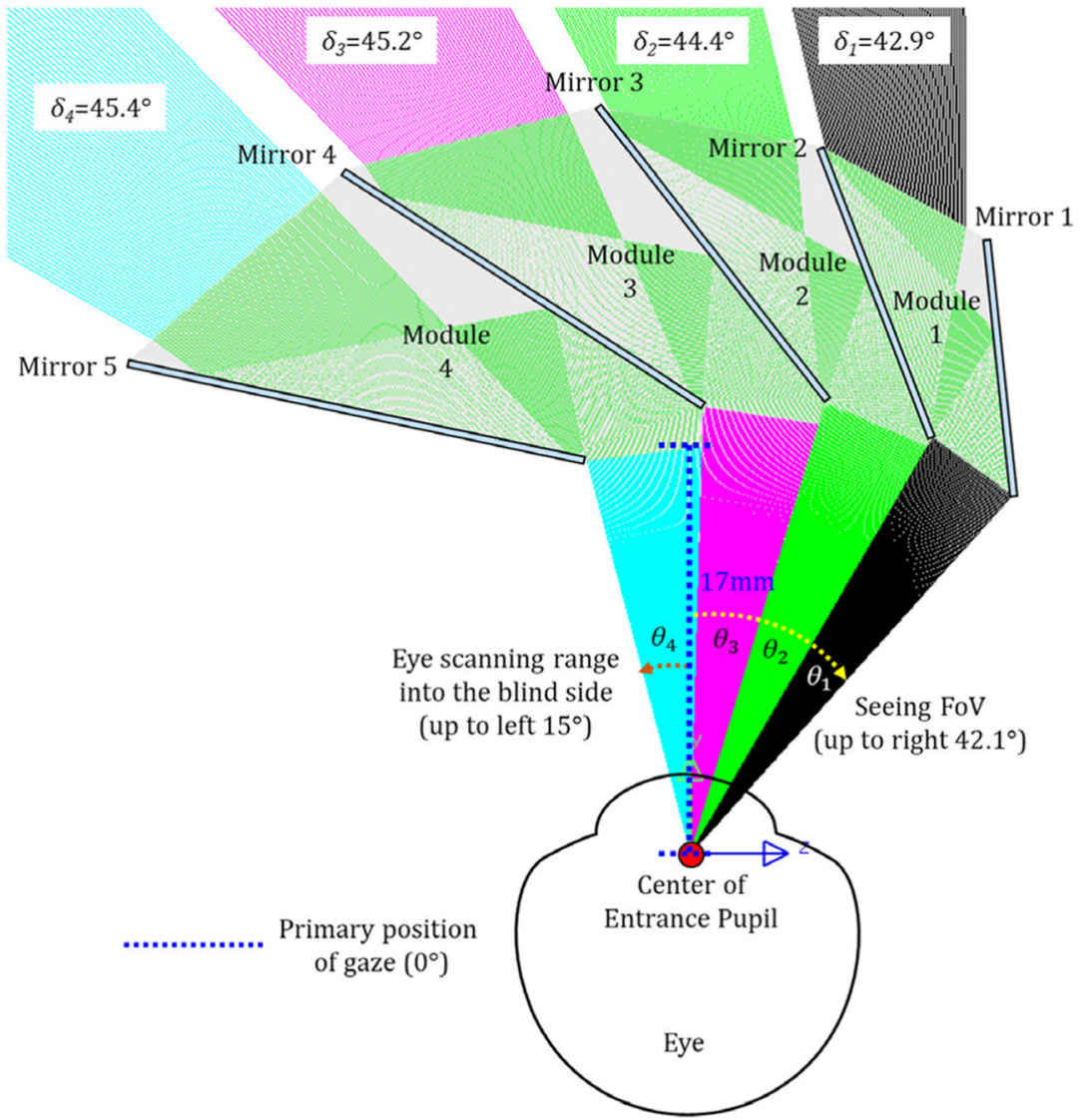

Fig. 5.

Ray tracing of the new MP design for left HH. Different colors mark rays in each module $\left(\sim 15^{\circ} \mathrm{FoV}\right)$. The deflection powers of the central ray in each MP module $\left(\delta_{n}\right)$ are matched with the designed values. Tunnel scotoma (gaps between ray bundles) is negligible in the practical distance. 
(a)

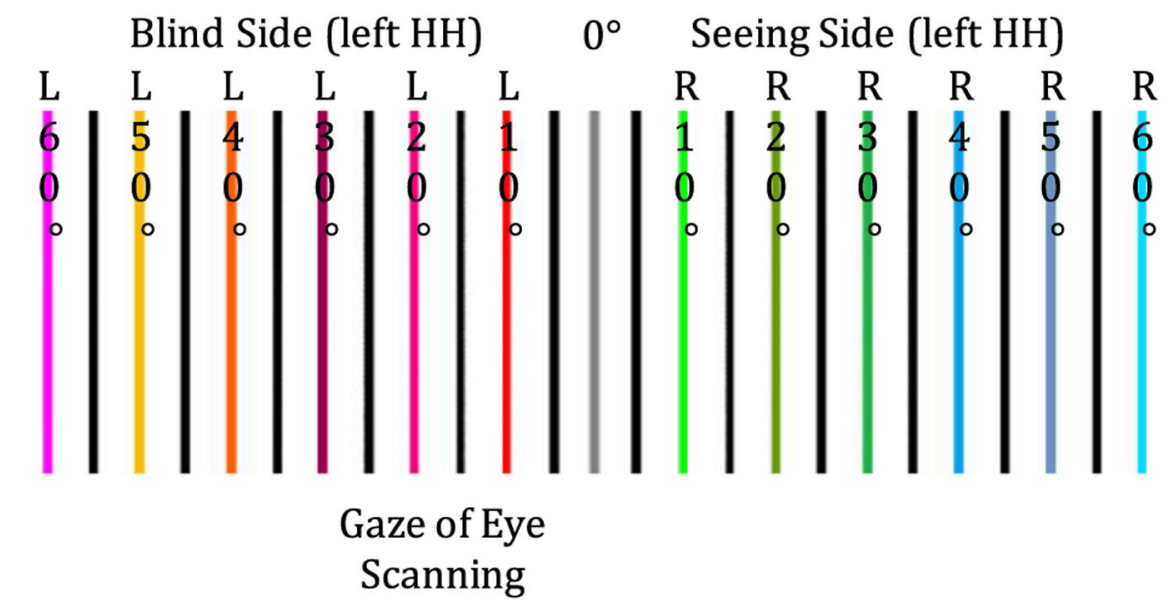

(b)

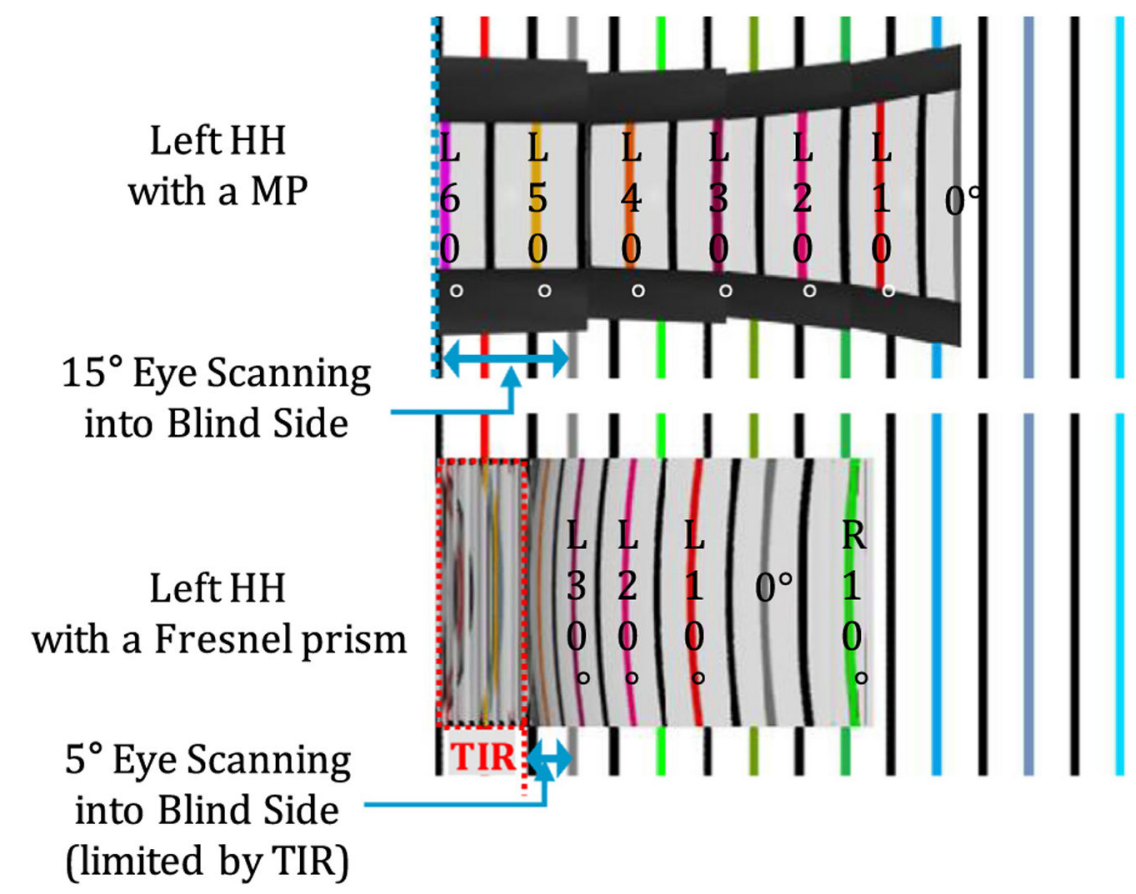

Fig. 6.

Simulated monocular scene with an MP and a Fresnel prism for a patient with left HH. (a) Target scene angular pattern with different color bars in $10^{\circ}$ steps and black bars in between. The side and eccentricities of the lines are labeled in degrees. (b) Perceived scene through the $100 \Delta \mathrm{MP}$. The MP was able to expand the visual field up to left $45^{\circ}$ from the primary position of gaze and up to $57^{\circ}$ with the $15^{\circ}$ eye scanning into the blind side. The image was almost free of distortion. The upper and lower black areas are scotoma caused by the protrusion of the MP. (c) Perceived scene through the conventional 57 $\Delta$ Fresnel prism. The conventional Fresnel prism provides field expansion of only $29^{\circ}$ at the primary position of gaze and limits eye scanning range into the blind side $\left(\sim 5^{\circ}\right)$ due to the TIR. Spurious reflection [4] and low image quality (distorted and dimmer) are seen in farther eccentricities. 
(a)

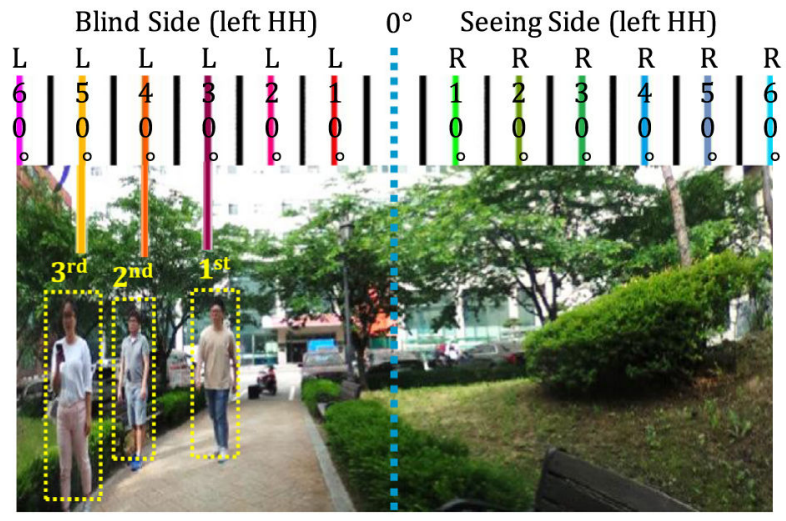

Gaze of Eye Scanning $0^{\circ}$
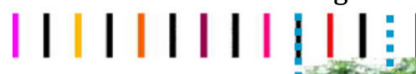

(b) $\quad \begin{gathered}\text { Left HH } \\ \text { with a MP }\end{gathered}$

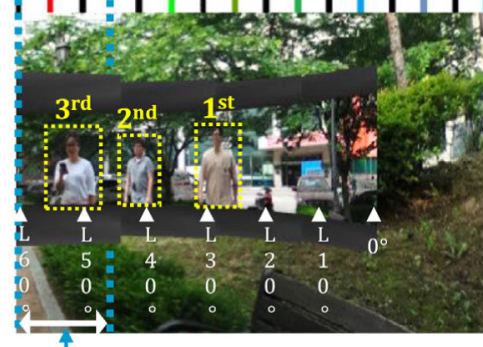

$15^{\circ}$ Eye Scanning into Blind Side

$0^{\circ}$

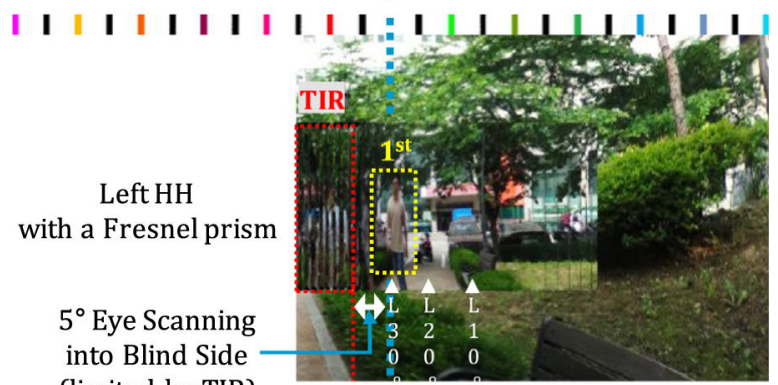

(limited by TIR)

Fig. 7.

Perceived scene of (a) a real scene with three pedestrians (at eccentricities of $29^{\circ}, 41^{\circ}$, and $51^{\circ}$ ). To indicate the angular location, we added the horizontal angular pattern (Fig. 6a) above the scene. (b) A left $\mathrm{HH}$ patient could detect the first two pedestrians (highest collision risk) at the primary position of gaze and the third pedestrian in the blind side through the $100 \Delta \mathrm{MP}$ with normal eye scanning. The upper and lower black areas are protrusion scotoma caused by the protrusion of the MP. (c) With the conventional $57 \Delta$ Fresnel prism, only the 1 st pedestrian would be detected in the blind side due to the prism distortion and TIR limitations. 
Table 1.

Specification of cascaded MP design with FoV of $57.1^{\circ}\left(42.1^{\circ}\right.$ in seeing FoV and $15^{\circ}$ for eye scanning range) and total deflection power of approximately $45^{\circ}$. Note that each module consists of parameters from two mirrors $(M$ and $D)$ and angular parameters between them $(\theta$ and $\delta$ ).

\begin{tabular}{ccccccc}
\hline Module & $\begin{array}{c}\boldsymbol{M} \\
(\mathbf{m m})\end{array}$ & $\begin{array}{c}\boldsymbol{D} \\
(\mathbf{m m})\end{array}$ & $\left.\boldsymbol{\theta}^{\circ}\right)$ & $\left.\boldsymbol{\delta}_{\boldsymbol{M}}{ }^{\circ}\right)$ & $\boldsymbol{\delta}_{\boldsymbol{R}}\left(^{\circ}\right)$ & $\left.\boldsymbol{\delta}^{\circ}\right)$ \\
\hline \multirow{2}{*}{1} & 10.7 & 20 & 12 & 30 & 12.9 & 42.9 \\
& 12.8 & 19.6 & & & & \\
2 & 12.8 & 19.6 & 13.5 & 33.8 & 10.6 & 44.4 \\
& 15.4 & 18.6 & & & & \\
3 & 15.4 & 18.6 & 15 & 37.5 & 7.7 & 45.2 \\
& 17.8 & 16.9 & & & & \\
4 & 17.8 & 16.9 & 16.6 & 41.5 & 3.8 & 45.4 \\
\hline
\end{tabular}

